\title{
Is the performance of a virtual sensor employed for the prediction of the ledge thickness inside a metallurgical reactor affected by the thermal contact resistance?
}

\author{
M. LeBreux, M. Désilets \& M. Lacroix \\ Faculté de génie, Université de Sherbrooke, Canada
}

\begin{abstract}
A virtual sensor is developed for predicting the time-varying thickness of the ledge on the inside surface of a wall of a high-temperature metallurgical reactor. The virtual sensor tracks the position of the solid-liquid phase front using thermal measurements taken from a heat flux sensor embedded in the reactor wall. The virtual sensor comprises a state observer coupled to a reduced model of the reactor. It also accounts for the thermal contact resistance of the wall structure. Results indicate that the virtual sensor is increasingly accurate as the magnitude of the thermal contact resistance augments. Moreover, the predictions of the virtual sensor remain accurate even when the contact resistance is poorly known.
\end{abstract}

Keywords: ledge, metallurgical reactor, virtual sensor, inverse method, thermal contact resistance, unscented Kalman filter, state-space model.

\section{Introduction}

The hostile conditions that prevail inside high-temperature metallurgical reactors (Figure 1) such as electric arc furnaces, blast furnaces and aluminum electrolysis cells [1-3], forbid the direct probing of the time-varying phase change protective layers that cover the inside surface of the walls. The standard method for monitoring these layers, hereupon called the ledge, is to probe it manually with a long metal rod [4]. This task is time consuming, inaccurate and risky. It also demands qualified personnel and measurements are usually taken days apart. 


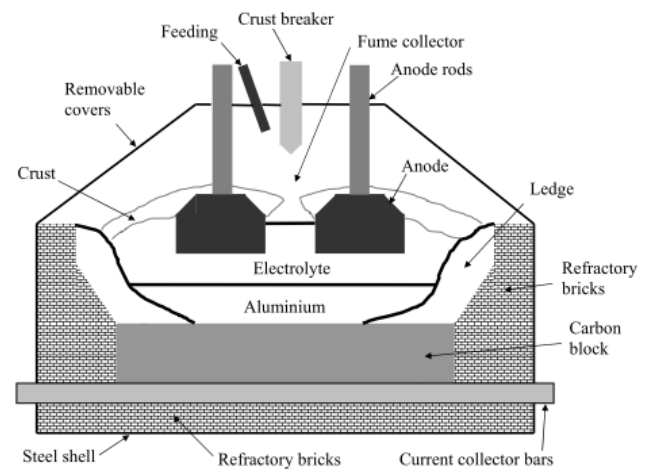

Figure 1: Cross-view of a typical metallurgical reactor.

Recently, LeBreux et al. have proposed a new non-invasive measurement technique for predicting the time-varying ledge thickness [5]. The technique rests on a nonlinear inverse heat transfer method that uses data collected by a thermal sensor embedded in the reactor wall (Figure 2). The inverse method corresponds to a virtual sensor that combines a nonlinear estimation algorithm (unscented Kalman filter) with thermal measurements (heat flux) thus enabling the on-line estimation of the process variable (ledge thickness).

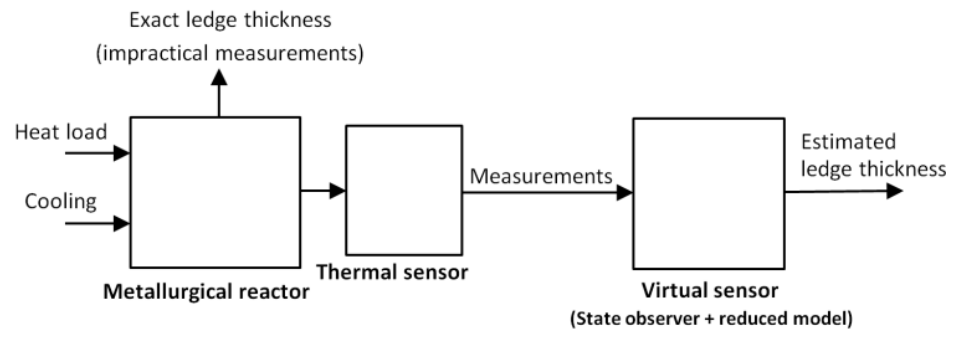

Figure 2: The virtual sensor for estimating the ledge thickness.

In this study, LeBreux et al. [5] have not taken into account the effect of the thermal contact resistance inside the wall structure. As a result, the response provided by the virtual sensor may not remain accurate for all operating conditions that prevail inside the furnace. Previous studies related to thermal contact resistances have focused on the estimation of its magnitude using inverse methods [7, 8]. To the authors' knowledge however, no study has shown the effect of the thermal contact resistance on the accuracy of the inverse prediction. The present study examines this question.

The paper is divided into four sections. First, the thermal model of a metallurgical reactor is presented. Second, a virtual sensor for estimating the ledge thickness is proposed. Third, the virtual sensor is thoroughly tested for typical operating conditions that prevail inside an industrial facility; the effect of the thermal contact resistance on the virtual sensor accuracy is exemplified. 
Finally, the virtual sensor is tested in cases for which the thermal contact resistance is poorly known. Its effect on the accuracy of the virtual sensor prediction is assessed.

\section{Thermal model of the metallurgical reactor}

The one-dimensional phase change problem under investigation is depicted in Figure 3. A time-varying ledge of thickness $s(t)$, which corresponds to the solidification front location, is built against the inner surface of a brick wall of thickness $L$. The outer surface of the reactor, a steel shell, is cooled by convection heat transfer with constant temperature $T_{\infty}$ and a heat transfer coefficient $h$. A thermal contact resistance $R$ ' contact is present in the wall structure between the steel shell and the brick wall. Finally, a time-varying heat flux $q$ " in $(t)$, which represents the heat load supplied to the reactor, is imposed on the right boundary condition at $x=L+D$.

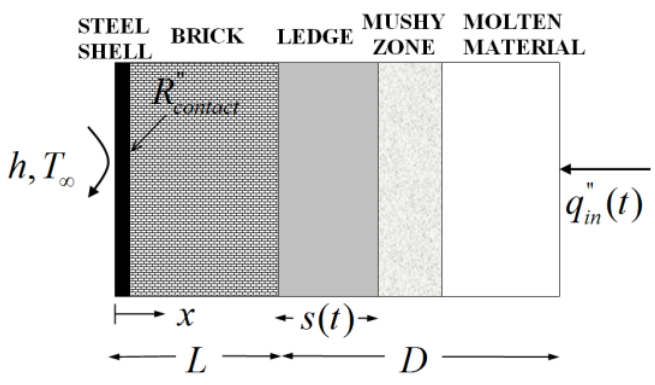

Figure 3: Schematic of the phase change direct problem.

The mathematical model for the phase change problem rests on the following assumptions:

- The FDM renders faithfully the thermal behavior of a real high-temperature metallurgical reactor $[1,9]$.

- The temperature gradients across the wall ( $x$ direction) are much larger than the temperature gradients in the vertical direction. As a result, a one-dimensional analysis can be applied $[1,9]$.

- The phase change problem is non-isothermal. It is characterized by a mushy zone between the solidus temperature $T_{\text {sol }}$ and the liquidus temperature $T_{\text {liq }}$.

- The thermal contact resistance $R$ " ${ }_{\text {contact }}$ between the steel shell and brick wall is taken into account by modifying the thermal conductivities of neighbouring materials.

- The thermal properties of the phase change material (PCM) are temperature independent; they may, however, be different for the liquid and the solid phase.

- The heat transfer across the liquid layer of the PCM is convection dominated. The effect of the flow circulation in the liquid layer is accounted by means of an effective thermal conductivity. 
The governing heat diffusion equation for the wall and the phase change material (PCM) may then be stated as:

$$
\rho C_{p} \frac{\partial T}{\partial t}=\frac{\partial}{\partial x}\left(k \frac{\partial T}{\partial x}\right)-\delta H \frac{\partial F}{\partial t}
$$

The boundary conditions are:

$$
\begin{gathered}
\left.k \frac{\partial T}{\partial x}\right|_{x=0}=h\left[T(0, t)-T_{\infty}\right] \\
\left.k \frac{\partial T}{\partial x}\right|_{x=L+D}=q^{\prime \prime}{ }_{i n}(t)
\end{gathered}
$$

the initial condition is:

$$
T(x, 0)=T_{0}(x)
$$

The volumetric enthalpy change $\delta H$ is defined as $\delta H=\rho\left(C_{p \text {,liquid }}-\right.$ $C_{p \text {,solid }} T+\rho \lambda$ where $\lambda$ corresponds to the latent heat of fusion of the PCM. The liquid fraction $F$ varies linearly between the solidus temperature $T_{\text {sol }}$ and the liquidus temperature $T_{\text {liq }}$ (Eq. 5).

$$
F=F(T)=\left\{\begin{array}{cc}
0 & T \leq T_{\text {sol }} \\
\frac{\left(T-T_{\text {sol }}\right)}{\left(T_{\text {liq }}-T_{\text {sol }}\right)} & T_{\text {sol }}<T<T_{\text {liq }} \\
1 & T \geq T_{\text {liq }}
\end{array}\right.
$$

Eqs. (1)-(5) were discretized using second-order finite differences in space, and first-order differences in time with an implicit scheme. The resulting set of algebraic equations is then solved using a tri-diagonal matrix algorithm (TDMA). For each time step, the liquid fraction $F$ in Eq. (1) is determined according to the enthalpy method, an iterative procedure developed by Voller and Swaminathan [10]. Thus, with the known conditions (Eqs. (2)-(5)), the FDM provides the evolution of the temperature field $T(x, t)$, the heat flux distribution $q$ ' $(x, t)$, and the ledge thickness $s(t)$.

The above finite-difference model (FDM) was thoroughly tested and validated using analytical solutions and results reported in the open literature. Further details concerning the validation of the FDM are provided in Ref. [5].

\section{Virtual sensor for predicting the ledge thickness}

In the inverse problem, the input heat flux $q{ }^{\prime \prime}(t)$ is unknown. Consequently, the ledge thickness $s(t)$ cannot be estimated directly (Figure 4). However, from thermal measurements $Y_{\text {mes }}(x, t)$ provided by a sensor embedded into the reactor's wall, it is possible to estimate $q{ }^{\prime \prime}{ }_{i n}(t)$ with a state observer and therefore to predict the time-varying ledge thickness $\hat{s}(t)$ using the reduced model of the metallurgical reactor. 


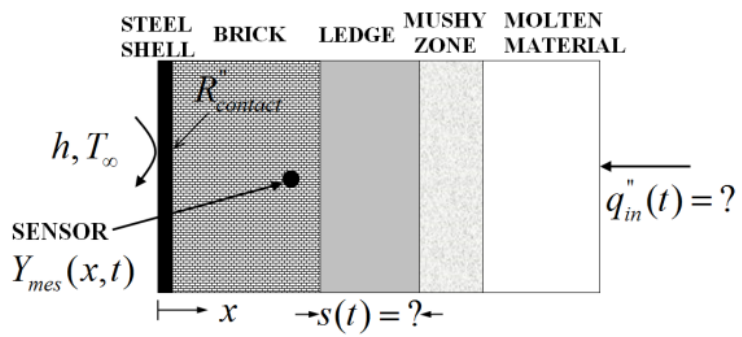

Figure 4: Schematic of the phase change inverse problem.

A state observer is a set of mathematical equations that provide an efficient recursive mean to estimate the unknown state of a process (the state vector). The most popular algorithm is the Kalman filter developed more than 50 years ago [11]. In order to estimate correctly the process state, the state observer proceeds through two steps: 1) the time update step (prediction) which provides an initial estimate of the state vector from the knowledge of the process dynamic model, followed by 2) the measurement update step (correction) that integrates sensor measurements in order to refine the state vector initial estimate.

As for the reduced model, the main objective is to extract, from knowledge of the reactor thermal model (section 2) a low-dimensional system (or a reduced model) that has nearly the same response characteristics as the process under study. The incentive behind model reduction is to come up with a simplified and computationally efficient model that captures the main features of the original complex model. Since the virtual sensor used in the current work is based on Kalman filtering, state-space models are more appropriate for building the reduced model.

The overall inverse methodology is summarized schematically in Figure 5. For more details on the mathematical formulation of the virtual sensor algorithm and on the reduced model, the reader is referred to Ref. [5]. In the current paper, two virtual sensors are compared for estimating the ledge thickness (Table 1). Each of them consists of a different combination of state observers and reduced models.

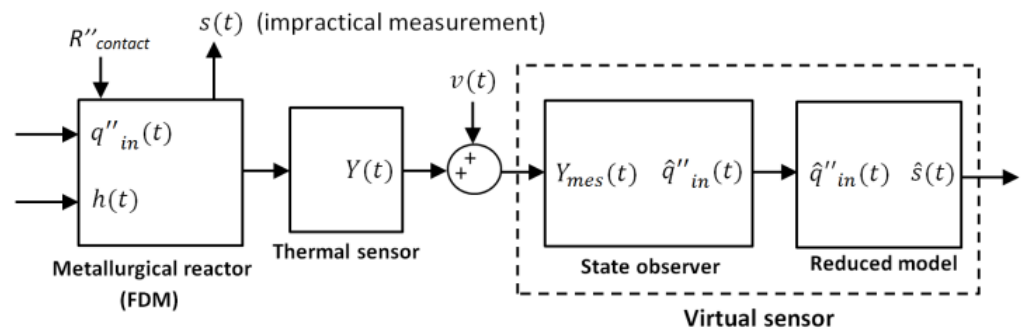

Figure 5: The virtual sensor for estimating the ledge thickness. 
Table 1: The virtual sensors.

\begin{tabular}{|c|c|c|c|c|}
\hline $\begin{array}{c}\text { Virtual } \\
\text { sensor }\end{array}$ & Type & State-observer & $\begin{array}{c}\text { Reduced } \\
\text { model }\end{array}$ & $\begin{array}{c}\text { Thermal } \\
\text { sensor }\end{array}$ \\
\hline$\# 1$ & Nonlinear & $\begin{array}{c}\text { Unscented } \\
\text { Kalman filter }\end{array}$ & $\begin{array}{c}\text { Nonlinear } \\
\text { state-space } \\
\text { model }\end{array}$ & $q^{\prime \prime}(x=L)$ \\
\hline$\# 2$ & Linear & $\begin{array}{c}\text { Classic Kalman } \\
\text { filter }\end{array}$ & $\begin{array}{c}\text { Linear state- } \\
\text { space model }\end{array}$ & $q^{\prime \prime}(x=L)$ \\
\hline
\end{tabular}

Even though its installation is cumbersome, the heat flux sensor is located at the brick/ledge interface $x=L$. The data collected by the virtual sensor at this location was found to be most accurate and yielded the lowest time lag as reported in Ref. [5]. The values of the covariance matrices $Q$ and $R$ (Table 2), which can be seen as the tuning factors of the virtual sensor, were chosen to achieve a compromise between the stability and the tracking capability of the inverse method. The stability refers to the oscillations in the solution triggered by the measurement noise, while the tracking capability refers to the time lag value, resulting from the heat diffusion phenomenon.

Table 2: Tuning factors of the virtual sensor.

\begin{tabular}{|c|c|}
\hline Covariance matrices & Values \\
\hline$Q$ & {$\left[\begin{array}{cc}0^{2} & 0 \\
0 & 0.01^{2}\end{array}\right]$} \\
\hline$R$ & $0.005^{2}$ \\
\hline
\end{tabular}

\section{Effect of the thermal contact resistance on the virtual sensor prediction}

Each of the virtual sensors were thoroughly tested for estimating the time-varying input heat flux $q{ }^{\prime}{ }^{\prime}$ on the right boundary and therefore for predicting the time-varying thickness of the ledge $s$ inside the reactor. For this reason, numerical simulations were carried out for typical operating conditions that prevail in such facilities [5]. In order to quantify the accuracy of the inverse prediction, the root-mean-square error $(R M S E)$ is adopted:

$$
R M S E=\sqrt{\frac{1}{N} \sum_{k=1}^{N}[s(k)-\hat{s}(k)]^{2}}
$$

where $k$ is the sampling instant and $N$ the total number of time steps.

The effect of the thermal contact resistance on the virtual sensor accuracy is presented in Table 3. First, one can note that the nonlinear virtual sensor \#1 provides a more accurate prediction than the linear virtual sensor \#2, due to the fact that the thermal model of the reactor is highly nonlinear. Moreover, it can be seen that for both virtual sensors, the $R M S E$ value decreases as $R$ "' contact increases. In other words, the virtual sensor prediction becomes more accurate as the thermal contact resistance increases. 
Table 3: The effect of $R{ }^{\prime \prime}{ }_{\text {contact }}$ on the accuracy of the virtual sensor.

\begin{tabular}{|c|c|c|}
\hline $\begin{array}{c}\boldsymbol{R}^{\prime \prime} \text { contact } \\
\left(\mathbf{m}^{\mathbf{2}} \mathbf{. K} / \mathbf{W}\right)\end{array}$ & $\boldsymbol{R M S E}$ Virtual sensor \#1 (m) & $\boldsymbol{R M S E}$ Virtual sensor \#2 (m) \\
\hline 0 & 0.0090 & 0.0090 \\
\hline 0.006 & 0.0069 & 0.0080 \\
\hline 0.010 & 0.0061 & 0.0078 \\
\hline 0.015 & 0.0058 & 0.0077 \\
\hline
\end{tabular}

For a small $R$ "' ${ }_{\text {contact }}$ (Figure 6), the ledge is thick. The thermal resistance increases and the time lags are prolonged and, as a result, the accuracy of the estimated ledge thickness is affected. On the other hand, when $R$ "' ${ }_{\text {contact }}$ is large (Figure 7), the ledge is thin. The inverse prediction becomes more accurate since the thermal resistance across the ledge and the time lags are small.

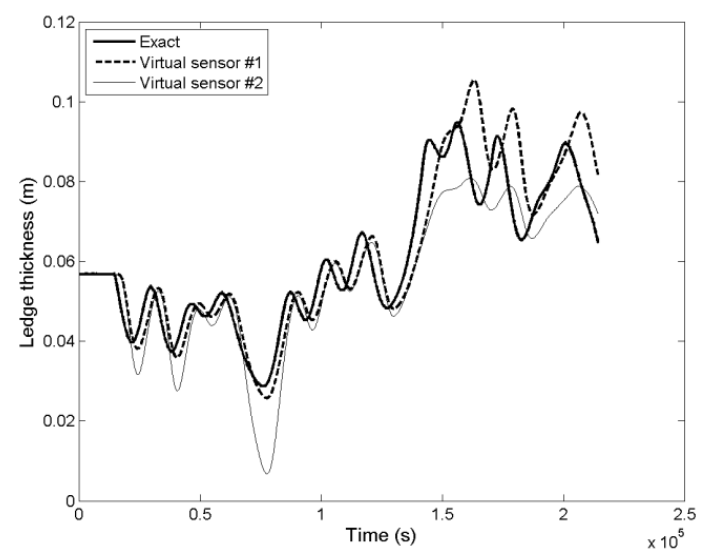

Figure 6: Exact and estimated ledge thicknesses $-R{ }^{\prime}{ }_{\text {contact }}=0 \mathrm{~m}^{2} . \mathrm{K} / \mathrm{W}$.

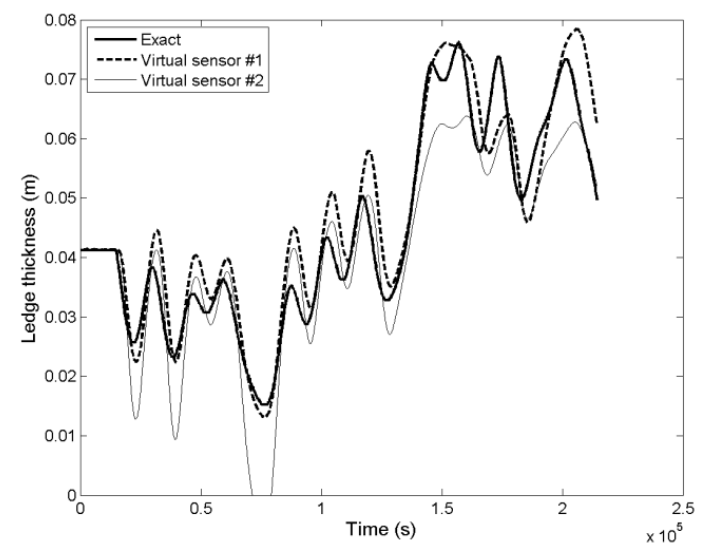

Figure 7: Exact and estimated ledge thicknesses $-R{ }^{\prime}{ }_{\text {contact }}=0.015 \mathrm{~m}^{2} . \mathrm{K} / \mathrm{W}$. 


\section{What if the thermal contact resistance is poorly known?}

The purpose of this section is to determine the performance of the virtual sensor when it is employed in a metallurgical reactor for which the thermal contact resistance is poorly known. The problem is depicted in Figure 8. The thermal contact resistance of the metallurgical reactor wall, known as the exact value, differs from the one assumed by the virtual sensor. As a result, the thermal behaviour of the metallurgical reactor will differ from that of the reduced model used by the virtual sensor, thereby challenging the performance of the inverse predictor.

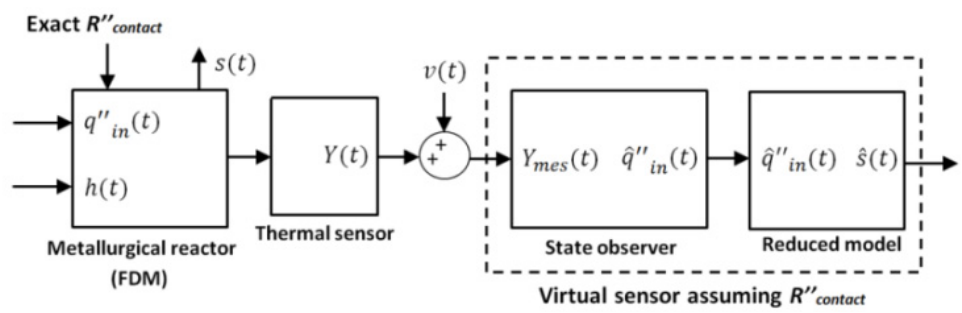

Figure 8: Does the virtual sensor remain accurate when $R_{\text {contact }}$ is poorly known?

To assess the performance of the virtual sensor in such a case, the RMSE value is invoked one more time (Eq. 6). Numerical simulations were thus carried out once again for typical operating conditions that prevail in such facilities. The results are presented in the form of four scenarios (Table 4). Scenarios \#1 and \#4 correspond to the cases studied in the previous section for which $R$ "' ${ }_{\text {contact }}$ is known. However, scenarios \#2 and \#3 represent the cases for which the virtual sensor overestimates and underestimates $R$ "' ${ }_{\text {contact }}$ respectively.

Table 4: $R$ ' ${ }_{\text {contact }}$ knowledge on the accuracy of the virtual sensor

\begin{tabular}{|c|c|c|c|c|}
\hline Scenario & $\begin{array}{c}\text { Exact } \\
R^{, \prime} \text { contact } \\
\left(\mathbf{m}^{2} . \mathbf{K} / \mathbf{W}\right) \\
\end{array}$ & $\begin{array}{c}\text { Assumed } \\
R^{\prime,} \text { contact } \\
\left(\mathbf{m}^{2} . \mathbf{K} / \mathbf{W}\right) \\
\end{array}$ & $\begin{array}{cc}R M S E & \text { Virtual } \\
\text { sensor \#1 (m) }\end{array}$ & $\begin{array}{c}R M S E \\
\text { Virtual sensor } \\
\# 2(\mathrm{~m}) \\
\end{array}$ \\
\hline$\# 1$ & 0 & 0 & 0.0090 & 0.0090 \\
\hline$\# 2$ & 0 & 0.015 & 0.0065 & 0.0089 \\
\hline$\# 3$ & 0.015 & 0 & 0.0062 & 0.0079 \\
\hline$\# 4$ & 0.015 & 0.015 & 0.0058 & 0.0077 \\
\hline
\end{tabular}

Table 4 reveals that when the virtual sensor overestimates $R$ '" ${ }_{\text {contact }}$ (scenario \#2), the inverse prediction becomes more accurate (scenario \#1). This can also be seen by comparing the exact and the estimated ledge thicknesses of Figure 9 with those of Figure 7. 


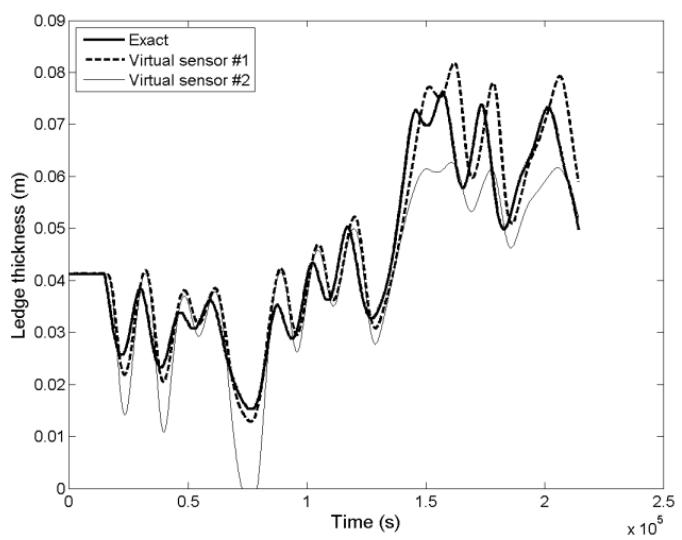

Figure 9: Exact and estimated ledge thicknesses - Scenario \#2.

On the other hand, when the virtual sensor underestimates $R$ ' ${ }_{\text {contact }}$ (scenario \#3), the inverse prediction is less accurate (scenario \#4). This is due to the larger thermal contact resistance of the metallurgical reactor.

Finally, one may note that the virtual sensor \#1 based on the unscented Kalman filter is much more sensitive to a poor knowledge of $R^{\text {' }}$ contact as the $R M S E$ variation in this case is much larger than that of the virtual sensor $\# 2$.

\section{Concluding remarks}

A virtual sensor was developed for predicting the time-varying thickness of the ledge on the inside surface of a wall of a high-temperature metallurgical reactor. The virtual sensor tracks the position of the solid-liquid phase front using thermal measurements taken from a heat flux sensor embedded in the reactor wall. The virtual sensor comprises a state observer coupled to a reduced model of the reactor. It also accounts for the thermal contact resistance the wall structure. Results have shown that the virtual sensor is increasingly accurate as the magnitude of the thermal contact resistance augments. Moreover, the predictions of the sensor remain accurate even when the contact resistance is poorly known.

\section{Acknowledgement}

The authors are very grateful to the Natural Sciences and Engineering Research Council of Canada (NSERC) for their financial support.

\section{References}

[1] O. Tadrari, M. Lacroix, Prediction of Protective Banks in HighTemperature Smelting Furnaces by Inverse Heat Transfer, International Journal of Heat and Mass Transfer 49 (13-14) (2006) 2180-2189. 
[2] J. Brännbacka, H. Saxén, Model for Fast Computation of Blast Furnace Hearth Erosion and Buildup Profiles, Ind. Eng. Chem. Res. 47 (2008) 7793-7801.

[3] M.P. Taylor, B.J. Welch, M.J. O'Sullivan, Sidewall Ledge Dynamics in Cells Used for Electrowinning Aluminium, Proceedings of the Eleventh Australian Conference on Chemical Engineering, 1983, pp. 493-500.

[4] K. Torklep, T. Nordbo, Some Applications of the Elkem Position Probe, Proceedings of the Minerals, Metals \& Materials Society (TMS), TMS, San Francisco, CA, 1994, pp. 449-456.

[5] M. LeBreux, M. Désilets, M. Lacroix, an Unscented Kalman Filter Inverse Heat Transfer Method for the Prediction of the Ledge Thickness Inside High-Temperature Metallurgical Reactors, International Journal of Heat and Mass Transfer 57 (1) (2013) 265-273.

[6] M. Kano, M. Ogawa, The State of the Art in Chemical Process Control in Japan: Good Practice and Questionnaire Survey, Journal of Process Control 20 (2010) 969-982.

[7] C. Fieberg, R. Kneer, Determination of Thermal Contact Resistance from Transient Temperature Measurements, International Journal of Heat and Mass Transfer 51 (5-6) (2008) 1017-1023.

[8] T. Loulou, E.A. Artyukhin, J.P. Bardon, Estimation of Thermal Contact Resistance during the First Stages of Metal Solidification Process. I. Experiment Principle and Modelisation, International Journal of Heat and Mass Transfer 42 (12) (1999) 2119-2127.

[9] C.C. Wei, J.J.J. Chen, B.J. Welch, V.R. Voller, Modelling of dynamic ledge heat transfer, Proceedings of the Minerals, Metals \& Materials Society (TMS), TMS, Orlando, FL, 1997, pp. 309-316.

[10] V.R. Voller, C.R. Swaminathan, General Source-Based Method for Solidification Phase Change, Numerical Heat Transfer 19 (1991) 175-189.

[11] R.E. Kalman, A New Approach to Linear Filtering and Prediction Problems, ASME Journal of Basic Engineering 82 (1960) 35-45. 